\title{
Shape Controllable Synthesis of ZnO Hexagonal Arrays via Chemical Precipitation
}

\author{
A. Medina-Flores*, L. Béjar-Gómez*, Gabriel Herrera-Pérez**, J.L. Bernal***. \\ *UMSNH, Ciudad Universitaria, Morelia, Michoacán. 58000. México. \\ **Departamento de Ingeniería en Materiales, Instituto Tecnológico Superior de Irapuato (ITESI), \\ Carretera Irapuato-Silao Km. 12.5, Irapuato, Guanajuato, México, C.P. 36821. \\ ***Universidad Politécnica del Valle de México. Avenida Mexiquense s/n. C.P. 54910. Tultitlán \\ Estado de México.
}

In recent years, the syntheses of nanoparticles with nanoscale structures have generated the most excitement in advanced-material research because of their fascinating properties from both scientific and engineering-oriented standpoints. For example due to the quantum confinement arising from the reduced dimensions of $\mathrm{ZnO}$ nanoparticles. In addition to the aforementioned optoelectric application, $\mathrm{ZnO}$ has also been used in electro-acoustic transducers, solid-state gas sensors, solar cells, transparent electrodes, photocatalyst and varistors [2]. The miniaturization of these devices also necessitates nanometer-sized $\mathrm{ZnO}$ structures.

The synthesis of bimetallic particles was made by a chemical precipitation method. Structural characterization of the samples was made using a FEG TEM TECNAI F20 microscope with analytical equipments attached. The samples were prepared by spreading a droplet of colloidal solution of $\mathrm{ZnO}$ nanoparticles onto a carbon film supported by a $\mathrm{Cu}$ grid and subsequent drying in vacuum. Samples were analyzed by high resolution microscopy and the elemental composition analysis was performed. The high resolution electron microscopy (HREM) images were processed by digital methods in real and Fourier spaces. Figure 1 shows a small cluster with sizes of 2 to $10 \mathrm{~nm}$ of range, the FFT image was taken of a small cluster showing an image of a hexagonal-like structure with a $0.27 \mathrm{~nm}$ of interatomic distance. It is likely that both elements are in the observed region as can be seen in the figure 2 .

\section{References}

[1] M.H. Huang et al. Science 292. (2001) 1897.

[2] I.V. Kityk, et al. Phys. Stat. Sol. 234 (2002) 553.

[3] Lingna Wang et al. Journal of Materials Chemistry, 9, No. 11, (1999). 2871-2878.

[4] HaiYan Xu et al. Ceramics International, Vol. 30, No. 1 (2004) pp. 93-97, 2004.

[5] Ning Wang et al. Crystal Research and Technology, Vol. 44, No. 3 (2009) 341-345. 

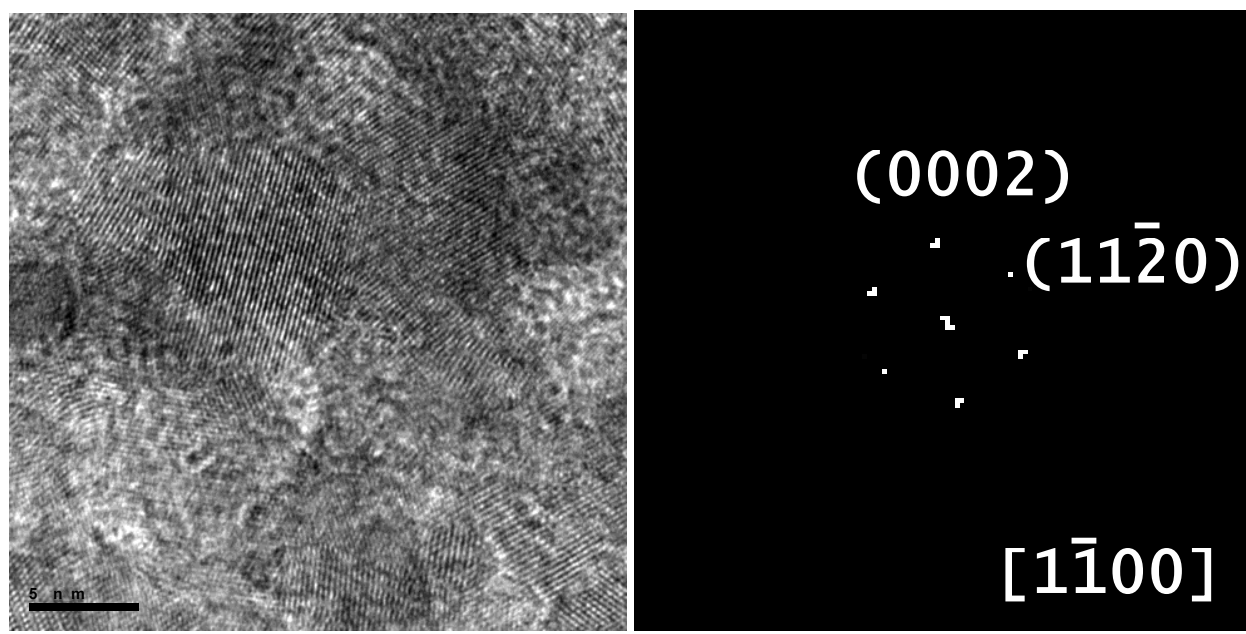

Figure 1: HRTEM image of a hexagonal-like structure with its corresponding FFT pattern.

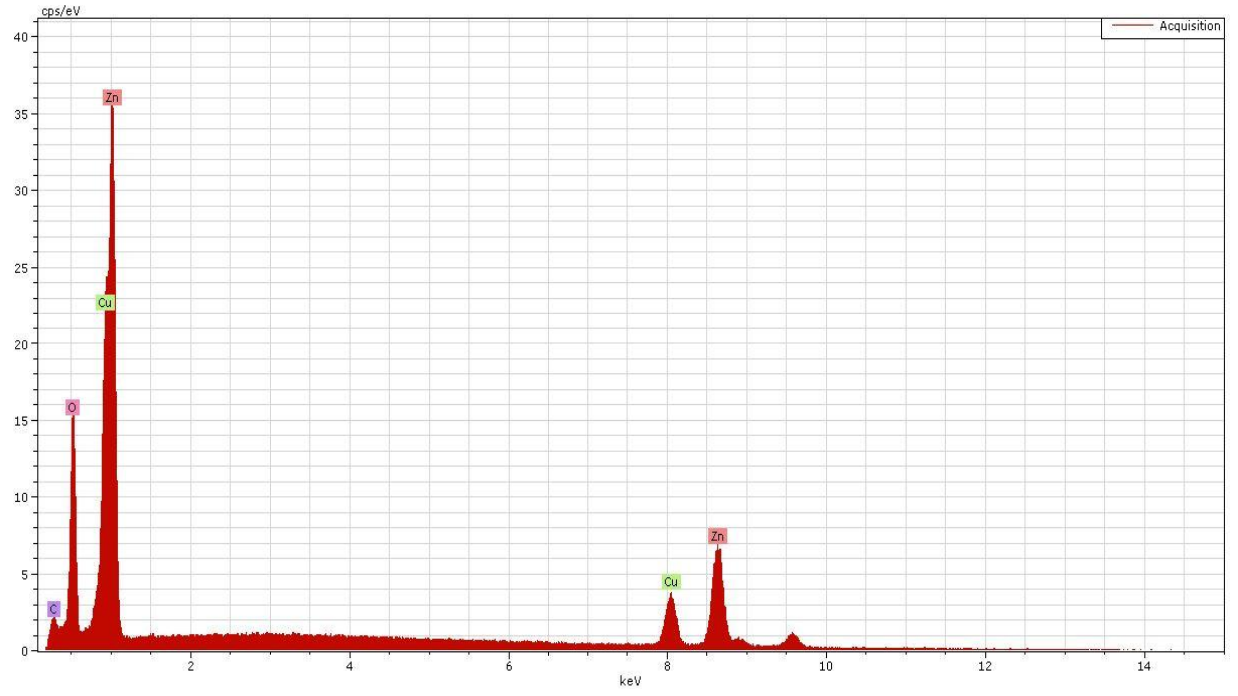

Figure 2: EDS spectrum of the $\mathrm{ZnO}$ nanoparticles 